\title{
Image Watermarking in the Linear Canonical Transform Domain
}

\author{
Bing-Zhao Li and Yu-Pu Shi \\ School of Mathematics and Statistics of Beijing Institute of Technology, Beijing 100081, China \\ Correspondence should be addressed to Bing-Zhao Li; li_bingzhao@bit.edu.cn
}

Received 26 December 2013; Revised 19 February 2014; Accepted 19 February 2014; Published 24 March 2014

Academic Editor: Juan J. Trujillo

Copyright (C) 2014 B.-Z. Li and Y.-P. Shi. This is an open access article distributed under the Creative Commons Attribution License, which permits unrestricted use, distribution, and reproduction in any medium, provided the original work is properly cited.

The linear canonical transform, which can be looked at the generalization of the fractional Fourier transform and the Fourier transform, has received much interest and proved to be one of the most powerful tools in fractional signal processing community. A novel watermarking method associated with the linear canonical transform is proposed in this paper. Firstly, the watermark embedding and detecting techniques are proposed and discussed based on the discrete linear canonical transform. Then the Lena image has been used to test this watermarking technique. The simulation results demonstrate that the proposed schemes are robust to several signal processing methods, including addition of Gaussian noise and resizing. Furthermore, the sensitivity of the single and double parameters of the linear canonical transform is also discussed, and the results show that the watermark cannot be detected when the parameters of the linear canonical transform used in the detection are not all the same as the parameters used in the embedding progress.

\section{Introduction}

Over the past several decades, digital watermarking become more and more important in the application of copyright protection for digital media as image, video, and audio [1-3]. A digital watermark is a code which embeds copyright information including sequence number, a picture, and text into the multimedia for copyright protection. The watermark must be easily detected by the copyright owner, the creator of the work, and the authorized consumer while is hardly read by the people who want to counterfeit the copyright of the data without authorization. Digital watermarking is an emerging technology in signal processing and communications which is under active development. The methods used to embed the watermark influence both the robustness and the detection algorithm. One of the hottest directions of the watermarking method is the watermarking in the transform domain, for example, in the discrete Fourier transform (DFT) domain [46] and in the discrete cosine transform (DCT) domain [7, 8], and the watermark proposed in [7] is two Gaussian sequences and it is embedded in the magnitude of the DCT transformation coefficients. A wealth of information and references can be found on the site of Watermarking World [9].
Recently, with the development of the fractional signal and processing technologies, the research results of the fractional Fourier transform (FRFT) and fractional Fourier operators have shown that the fractional domain signal processing can be looked at as one of the hottest research topics for nonstationary signals processing [10-15]. Several digital watermarking methods are proposed in the FRFT Domain [16-19] base on these novel results of the FRFT. A nonsensical watermark embedded in the FRFT domain was proposed in [16], and it has a more security because of the free parameter of the FRFT. Bultheel [18] describes the implementation of a watermark embedding technique in the FRFT domain in detail and also discusses the embedding several watermarks at the same time for images. The practical detecting threshold proposed in [18] is one of the most important contributions of the paper. All of these results, which come from the digital watermarking technology in the FRFT domain, have shown that the watermarking method in these transform domains can be more secure and hard to be detected compared to the traditional method in the classical DFT and DCT domain.

The linear canonical transform (LCT) [20], which can be looked at as the further generalization of the fractional 
Fourier transform, is introduced in the 1970s with three free parameters and has been proven to be one of the most powerful tools for nonstationary signal processing. The wellknown signal processing operations, such as the Fourier transform (FT), the FRFT, the Fresnel transform, and the scaling operations, are all special cases of the LCT [20]. The digital computation methods of the LCT have been proposed in [21-24], and the sampling theories associated with the LCT have been studied in [25-29], and the eigenfunction [30], the convolution and product function [31, 32], and the uncertainty principle [33] have also been investigated in detail. Therefore, understanding the LCT may help to gain more insight into its special cases and to carry the knowledge gained from one subject to others [20].

However, for the best of our knowledge, there are no papers published about the watermarking in the LCT domain. So it is interesting and worthwhile to investigate the watermarking method and technique associated with the LCT. Focusing on this problem, a novel watermarking technique based on the discrete LCT proposed in [23] is proposed in this paper. The experiment results show that the embedded watermarks are both perceptually invisible and robust to various image processing techniques. The remaining of this paper can be divided into the following sections. The LCT is described in Section 2. Section 3 develops watermark embedding in LCT domain. Numerical examples and the discussion of the simulation results are given in Section 4 , and Section 5 is the conclusion.

\section{The Linear Canonical Transform}

2.1. The Continuous LCT. The continuous LCT of a signal $f(x)$ with parameter matrix $A=\left(\begin{array}{ll}a & b \\ c & d\end{array}\right)$ can be defined as [20]

$$
\begin{gathered}
f_{A}(y)=C_{A}(f)(y)=\int_{-\infty}^{+\infty} f(x) C_{A}(x, y) d x, \\
C_{A}(x, y) \\
=\sqrt{\frac{1}{b}} e^{-j \pi / 4} \exp \left\{j \pi\left[\left(\frac{a}{b}\right) x^{2}-\left(\frac{2}{b}\right) x y+\left(\frac{d}{b}\right) y^{2}\right]\right\},
\end{gathered}
$$

where $C_{A}$ is the LCT operator and $a, b, c, d$ are real parameters. Furthermore the constraint $a d-b c=1$ must be satisfied to make the transform unitary. Actually the LCT has three free parameters; if we let $a=\gamma / \beta, b=1 / \beta, c=-\beta+\alpha \gamma / \beta$, $d=\alpha / \beta$, the LCT of $f(x)$ can be rewritten as [23]

$$
\begin{gathered}
f_{A}(y)=C_{A}(f)(y)=\int_{-\infty}^{+\infty} f(x) C_{A}(x, y) d x, \\
C_{A}(x, y)=\sqrt{\beta} e^{-j \pi / 4} \exp \left[j \pi\left(\gamma x^{2}-2 \beta x y+\alpha y^{2}\right)\right],
\end{gathered}
$$

where parameter matrix

$$
A=\left(\begin{array}{ll}
a & b \\
c & d
\end{array}\right)=\left(\begin{array}{cc}
\frac{\gamma}{\beta} & \frac{1}{\beta} \\
-\beta+\frac{\alpha \gamma}{\beta} & \frac{\alpha}{\beta}
\end{array}\right) .
$$

Two of interesting and important properties of LCT are reversibility and index additivity. Index additivity means that, if two LCTs with matrices $A_{1}, A_{2}$ operate in a successive manner, then the equivalent transform is an LCT with the matrix $A=A_{1} A_{2}$. Because of the index additivity, the inverse of the LCT with matrix $A$ is an LCT with the matrix $A^{-1}$.

With the development of the fractional signal processing method, the properties and applications of the LCT have been investigated in detail; for more information associated with the continuous LCT, one can refer to $[14,15,20]$.

2.2. The Discrete LCT. Besides the continuous LCT, we often encounter the computation of the discrete LCT because we must process discrete data by computer. There are lots of discrete and the fast LCT methods proposed in the literature $[21,23,24]$. If we set $\delta_{x}=\delta_{y}=(N|\beta|)^{-1 / 2}, x=n \delta_{x}, y=m \delta_{y}$, and $m, n=0,1, \ldots, N-1$, the $N$ point discrete LCT (DLCT) of $f(n)$ can be defined as [23]

$$
f_{A}(m)=\sum_{n=0}^{N-1} f(n) C_{A}(m, n)
$$

where

$$
\begin{aligned}
& C_{A}(m, n) \\
& \quad=\frac{\sqrt{\beta} e^{-(j \pi / 4)}}{\sqrt{N|\beta|}} \exp \left[j \pi \frac{1}{N|\beta|}\left(\alpha m^{2}-2 \beta m n+\gamma n^{2}\right)\right] .
\end{aligned}
$$

This kind of DLCT method is available for image processing, because it is interval-independent and unitary. Moreover, it also has the property of index additivity.

Following this method, the two-dimensional DLCT of a size $H \times N$ image $I(h, n)$ can be rewritten as

$$
I_{A}(k, l)=\sum_{n=0}^{N-1} C_{A}(l, n) \sum_{h=0}^{H-1} I(h, n) C_{A}(k, m)
$$

with $k=0,1, \ldots, H-1, l=0,1, \ldots, N-1$, and $C_{A}(k, m)$, $C_{A}(l, n)$ being the same as (4). It is shown in [23] that this kind of DLCT is analogous to the DFT and approximates the continuous LCT in the same sense that the DFT approximates the continuous Fourier transform. We will use this method to compute the 2D LCT of an image in the following sections.

\section{Watermark Embedding and Detecting}

It is well known that the watermarking process contains the watermark embedding and detecting steps; we propose a new kind of watermarking scheme following the idea of [18] in this section.

3.1. Watermark Embedding. The watermark itself is a sequence of $M$ complex numbers [18], denoted by $s_{i}=c_{i}+j d_{i}$, $i=1,2, \ldots, M$, and the real and imaginary parts of $s_{i}$ are obtained from a normal distribution with mean zero 
and variance $\sigma^{2} / 2$. In order to embed this watermark into an image $I$ of size $H \times N$, we first computed the DLCT of this image $I$ to derive the transform coefficients $\left\{S_{i}: i=1 \times N\right\}$ and then reordered the transform coefficients in nonincreasing sequence as follows:

$$
S_{i}=C_{i}+j D_{i}:\left|S_{i}\right| \leq\left|S_{i+1}\right|, \quad i=1,2 \ldots H \times N .
$$

Similar with the method in [16], we chose the middle reordered transform coefficients to embed the watermarks; in other words, we embed the watermark into the coefficients $S_{i}, i=L+1, L+2, \ldots, L+M$. This is because if we embedded the watermarks in the lowest coefficients, they would be sensitive to noise removing or compressing operations, while if we embedded the watermarks in the highest coefficients, they would significantly affect the imperceptibility of the watermarks. So, the watermarks were embedded as follows:

$$
S_{i}^{w}=S_{i}+c_{i}\left|C_{i}\right|+j d_{i}\left|D_{i}\right|, \quad i=L+1, \ldots, L+M,
$$

where $S_{i}^{w}$ is the watermarked image of $I$ and $\left(c_{i}, d_{i}\right)$ is the watermarks sequence.

3.2. Watermark Detecting. When the watermark is embedded in the image, then the image is transferred to the watermark detection process to see whether it contains watermark. The detection of the watermark can be described like this: given the watermarked image $I^{a}$, maybe under some attacks such as low pass and median filtering, addition of Gaussian noise, and resizing, we compute the DLCT of $I^{a}$ and obtain the transform coefficients $S^{a}$ and then compute the detection value [16]:

$$
d=\sum_{i=L+1}^{L+M}\left(c_{i}-j d_{i}\right) S_{i}^{(a)} .
$$

The threshold can be achieved according to the statistical performance of the proposed algorithm. The expected value of $d$ is

$$
E[d]=\frac{\sigma^{2}}{2} \sum_{i=L+1}^{L+M}\left(\left|C_{i}\right|+\left|D_{i}\right|\right)
$$

In [16], Djurovic et al. propose a useful and simple threshold as $E[d] / 2$; when the value of $d$ is larger than the threshold, it is decided that a watermark has been detected. Otherwise, there is no watermark. However, it is shown in [18] that this kind of threshold suffers from the false conclusion; therefore we use an adaptive threshold proposed in [18], because it is more practical when we deal with the image after some attacks. Therefore, the threshold can be computed by the following steps.

(i) First, we compute the value of $d$ of all the random watermarks (maybe 1000 watermarks).

(ii) Then, we compute the average (say $\mu$ ) and the standard deviation (say $\sigma$ ) of these $d$.

(iii) At last, we can achieve the threshold $\tau=\mu+p \sigma$ where $p$ is a suitable number.

\section{Simulation Examples}

4.1. Watermark Embedding and Detecting. The Lena $(512 \times$ 512) was chosen as the test image in the simulations. According to some experiments, the value of $p$ in threshold $\tau=$ $\mu+p \sigma$ was chosen to be 5 . The 2D DLCT parameters are $\alpha_{1}=$ $\alpha_{2}=0.2, \beta_{1}=\beta_{2}=0.6, \gamma_{1}=\gamma_{2}=0.1$ and can be described as $\left(\alpha_{1}, \beta_{1}, \gamma_{1}, \alpha_{2}, \beta_{2}, \gamma_{2}\right)=(0.2,0.6,0.1,0.2,0.6,0.1)$. Therefore, the 2D DLCT parameter matrixes can be rewritten as

$$
A_{1}=A_{2}=\left(\begin{array}{cc}
\frac{\gamma}{\beta} & \frac{1}{\beta} \\
-\beta+\frac{\alpha \gamma}{\beta} & \frac{\alpha}{\beta}
\end{array}\right)=\left(\begin{array}{cc}
\frac{1}{6} & \frac{5}{3} \\
-\frac{17}{30} & \frac{1}{3}
\end{array}\right),
$$

and the 2D DLCT is performed based on (6). The simulations performed using Matlab version 7.5.0 in Windows 8 system and the processer of the system is Intel(R) Core(TM) i5$3337 \mathrm{U}$; the CPU and the RAM of the system are $1.80 \mathrm{GHz}$ and $4.00 \mathrm{~GB}$, respectively. We chose $L=96000, M=12000, \sigma^{2}=$ 60 in the simulation. In order to test the performance of the proposed method, we use the PSNR and the elapsed time of the process to measure the performance of the watermarking technology [18].

The original and watermarked images are shown in Figures 1(a) and 1(b), respectively. It is shown that the watermarked picture Figure 1(b) is almost the same as the original Figure 1(a). The detection of the correct watermark from the watermarked image over the other 1000 different watermarks, which are also Gaussian white noise with variance $\sigma_{G}^{2}=$ $\sigma^{2} / 2=30$. The detection result is plotted in Figure 2. In this case, the PSNR and the elapsed time are $39.27 \mathrm{~dB}$ and 16.147 seconds, respectively.

In Figure 2, we can easily find that the detection value of the correct watermark is significantly larger than the threshold and other false watermarks. So, the watermark can be detected by the comparison.

4.2. The Robustness. In this subsection, we investigate the robustness of the algorithm after the following attacks: adding noise, upper cropping, central cropping, and central cropping after adding noise. These experiments have been performed as the following.

Firstly, Figures 3 and 4 plot the robustness of the watermarking under the Gaussian noise. Figure 3(a) is the noisy image of the watermarked image in Figure 1(b) by adding mean zero and variance 200 Gaussian noise, while the variance of Figure 4(a) is 600. Figures 3(b) and 4(b) are detection results of these two situations, the PSNR are $19.76 \mathrm{~dB}$ and $15.08 \mathrm{~dB}$, the elapsed times are 9.75 and 26.82 seconds, respectively. This result shows that the method is robust against noise, because the watermark can be still detected.

Secondly, we cropped the watermarked image Figure 1(b) from the size $512 \times 512$ to $412 \times 212$ and $212 \times 212$, and obtain Figures 5(a) and 6(a), respectively. The detection results are shown in Figures 5(b) and 6(b), respectively. It is shown in Figures 5 and 6 that the watermark can also be detected. In this situation, the PSNR are $1.51 \mathrm{~dB}$ and $0.82 \mathrm{~dB}$, the elapsed time are 28.70 and 29.65 seconds, respectively. 


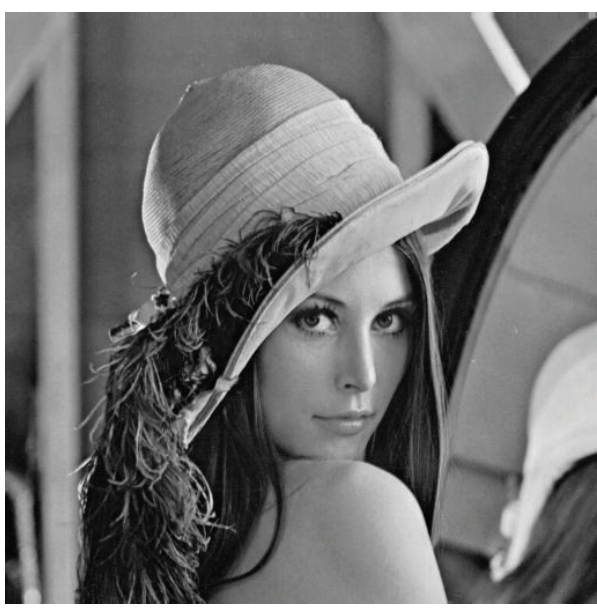

(a)

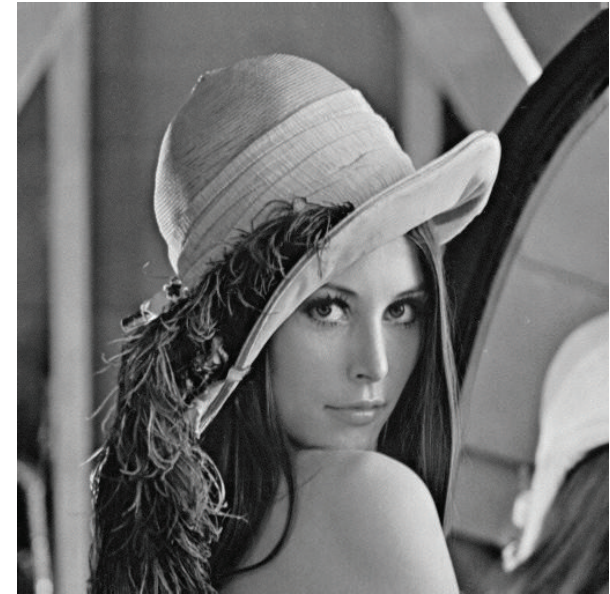

(b)

Figure 1: (a) The original image of "Lena", (b) the watermarked image of "Lena".

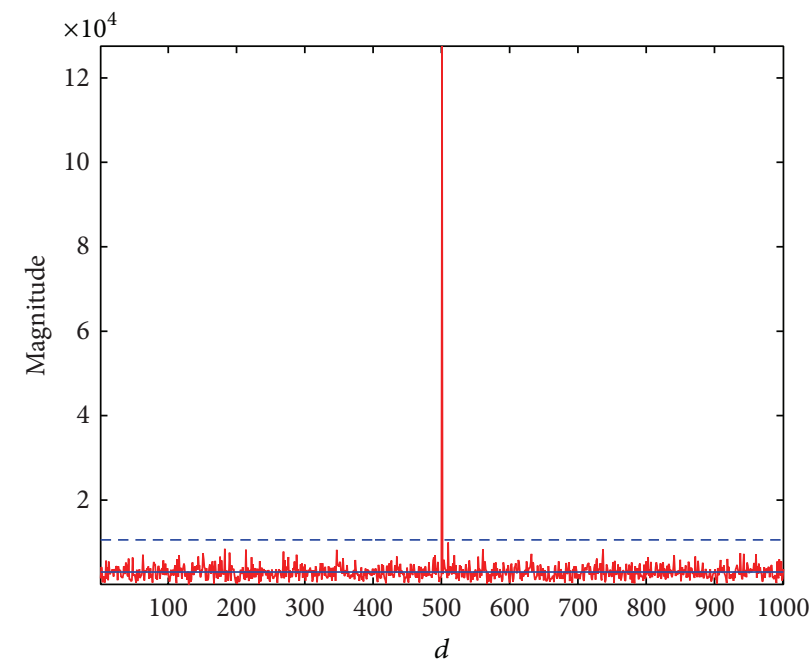

FIgURE 2: The detection result from the watermarked Figure 1(b).

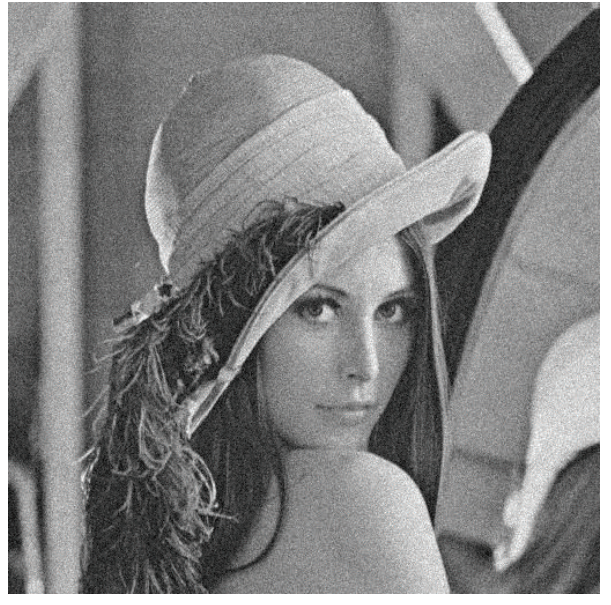

(a)

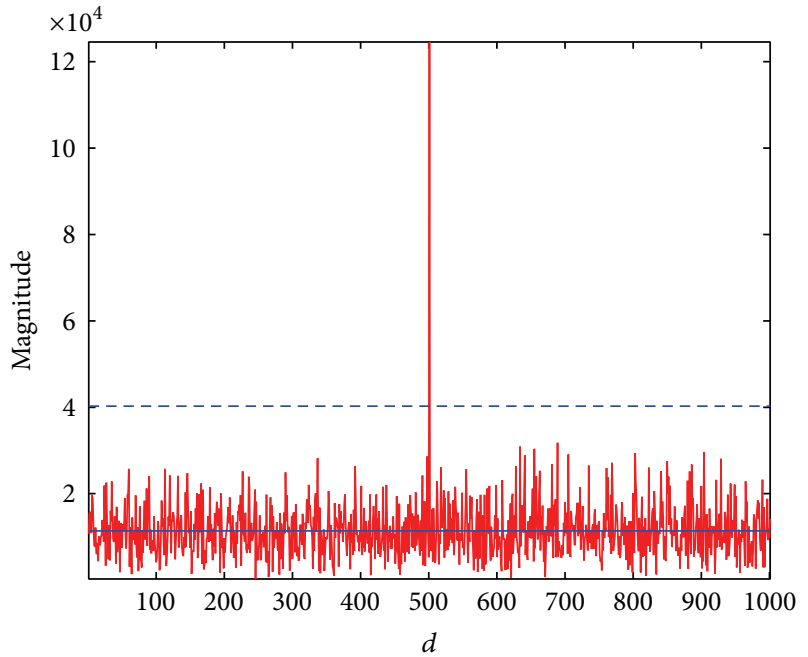

(b)

Figure 3: (a) The noisy "Lena," var = 200. (b) The detection of the noisy "Lena." 


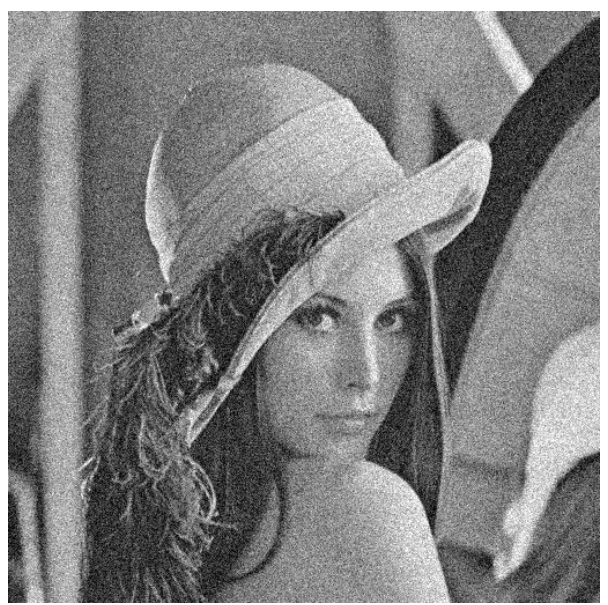

(a)

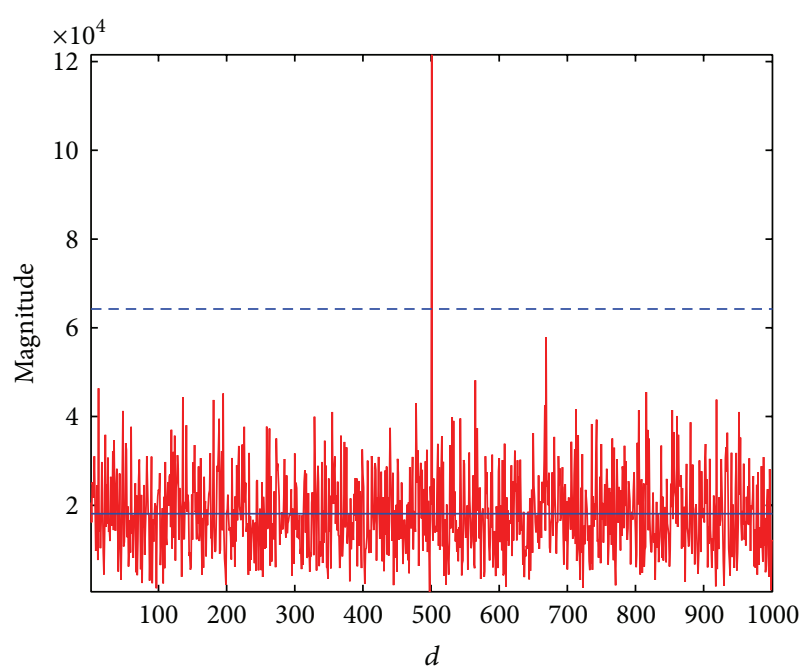

(b)

Figure 4: (a) The noisy "Lena," var $=600$. (b) The detection of the noisy "Lena."

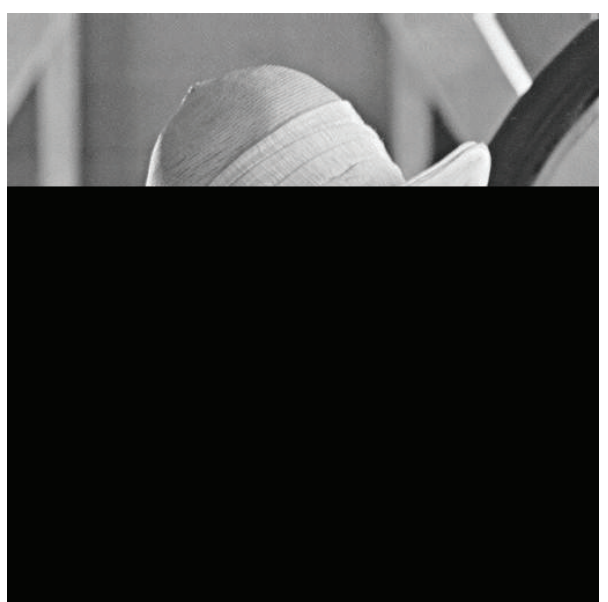

(a)

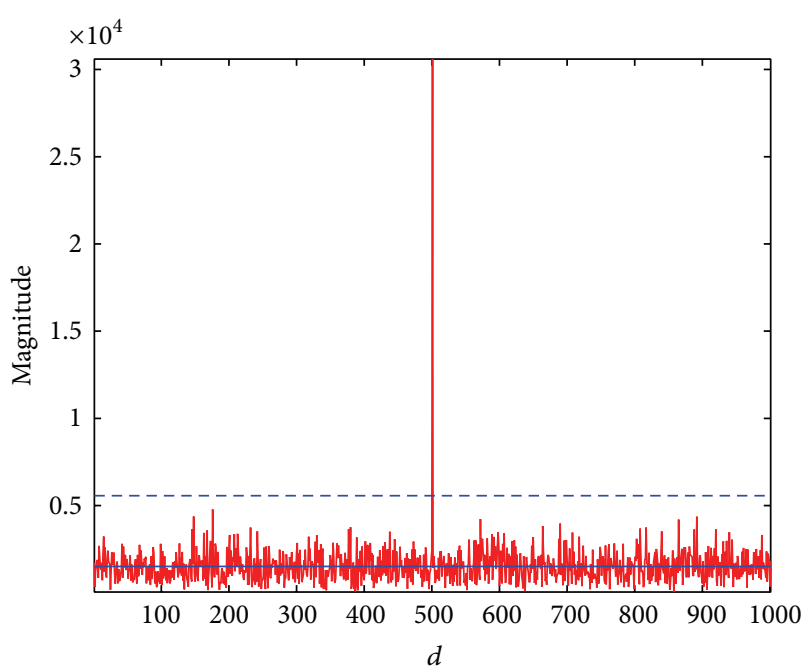

(b)

Figure 5: (a) The upper cropped image of Figure 1(b). (b) The detection of upcropped image.

Thirdly, we perform the upper cropping of the noisy image in Figures 3(a) and 4(a) in the same way as in Figure 5(a) and obtain Figures 7(a) and 8(a). The detection results are plotted in Figures 7(b) and 8(b), respectively. It is shown in Figure 7 that the watermark can also be detected for the upper cropped noisy watermarked image of variance 200 . We can still detect the watermark for the upper cropped noisy image of variance 600 as shown in Figure 8. In this situation, the PSNR are $1.50 \mathrm{~dB}$ and $1.48 \mathrm{~dB}$, and the elapsed times are 28.70 and 29.288 seconds, respectively.

Lastly, we central crop the noisy image in Figures 3(a) and 4(a) in the same way as in Figure 6(a) and obtain Figures 9(a) and 10(a). The detection results are plotted in Figure 9(b) and Figure 10(b), respectively. It is shown in Figure 9 that the watermark can also be detected for the central cropped noisy watermarked image of variance 200 . We can still detect the watermark for the central cropped noisy image of variance 600 as shown in Figure 10. In this situation, the PSNR are $0.8 \mathrm{~dB}$ and $0.78 \mathrm{~dB}$, and the elapsed times are 29.45 and 29.03 seconds, respectively.

From these simulations, it can be concluded that the proposed method is robust under the common image attacks, such as the noise, crops, and the crops of the noisy image. It should be also noticed from Figures 8 and 10 that the proposed method still works under the attack of cropping if the variance of the adding noise is about 600 . 


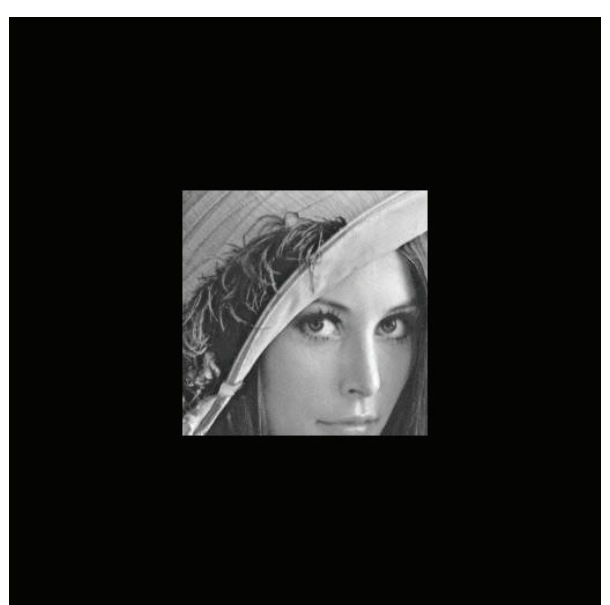

(a)

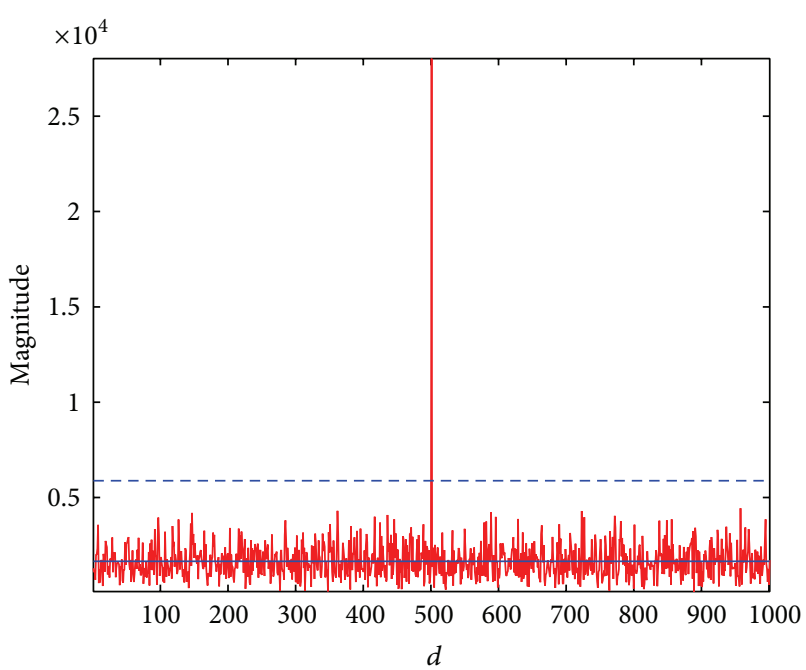

(b)

FIgURE 6: (a) The central cropped image of Figure 1(b). (b) The detection of central cropped image.

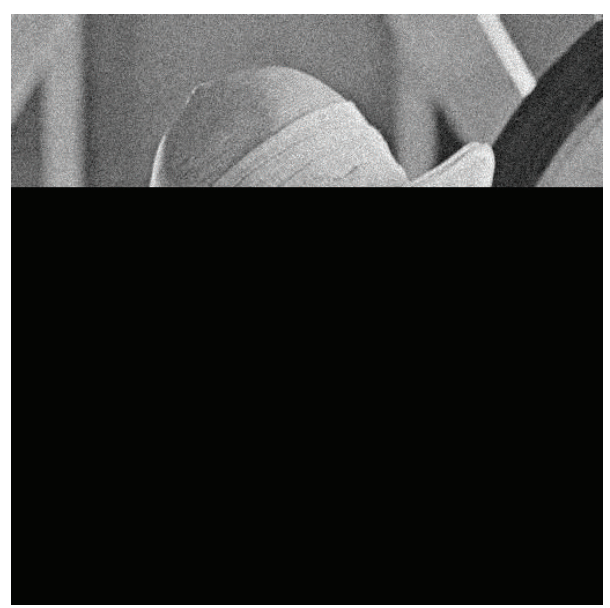

(a)

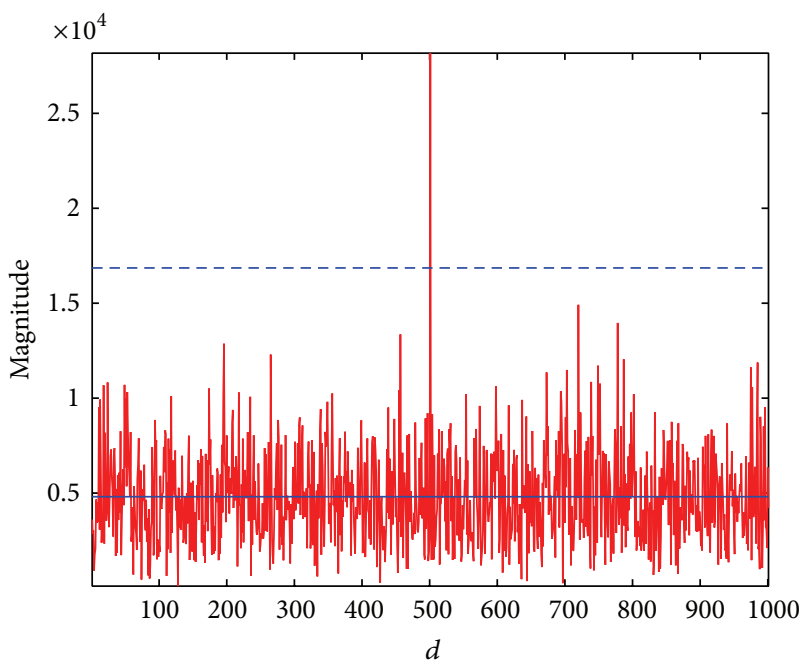

(b)

Figure 7: (a) The upper cropped noisy "Lena" of Figure 3(a). (b) The detection of the upcropped noisy "Lena."

4.3. The Parameters' Sensitivity. As compared to the traditional watermarking method, for example, the DFT and DCT domain method [5-8], the advantage of the proposed method is that it has three more free parameters, and this can enhance the security and robustness of the watermarking images. It is well known that the parameters of the LCT are two more than the parameters of the FRFT, and for the 2D-LCT there are six parameters. So, when we need to detect the watermarks, we not only need the watermarked keys but also need the six parameters which is three times the number of the FRFT's parameter. Therefore, it is more difficult for the unauthorized person to detect the watermark and destroy it.

In order to show the advantage of the LCT based watermarking method proposed in this paper, the sensitivity of the parameter $\left(\alpha_{1}, \beta_{1}, \gamma_{1}, \alpha_{2}, \beta_{2}, \gamma_{2}\right)$ is discussed in this subsection. We use the watermarked image in Figure 1(b) as tested image, we set $\left(\alpha_{2}, \beta_{2}, \gamma_{2}\right)=(0.2,0.6,0.1)$, and do not know the value of $\alpha_{1}, \beta_{1}$, and $\gamma_{1}$ in simulations; the value of $d$ is sensitive with the $\alpha_{1}, \beta_{1}$, and $\gamma_{1}$ as plotted in Figure 11 .

It is shown in Figure 11 that the value of $d$ is significantly larger when the value of $\alpha_{1}, \beta_{1}$, and $\gamma_{1}$ are more correct than the false values of the parameters. For example, when the unauthorized people know $\left(\beta_{1}, \gamma_{1}, \alpha_{2}, \beta_{2}, \gamma_{2}\right)=$ $(0.6,0.1,0.2,0.6,0.1)$, the correct place of the watermark, and the correct watermark but not sure about the value of $\alpha_{1}$, the watermark still cannot be detected because only the $d$ value of correct $\alpha_{1}$ can reach the peak according to Figure 11(a). We can also see that the sensitivity of $\alpha_{1}$ and $\beta_{1}$ is good, while 


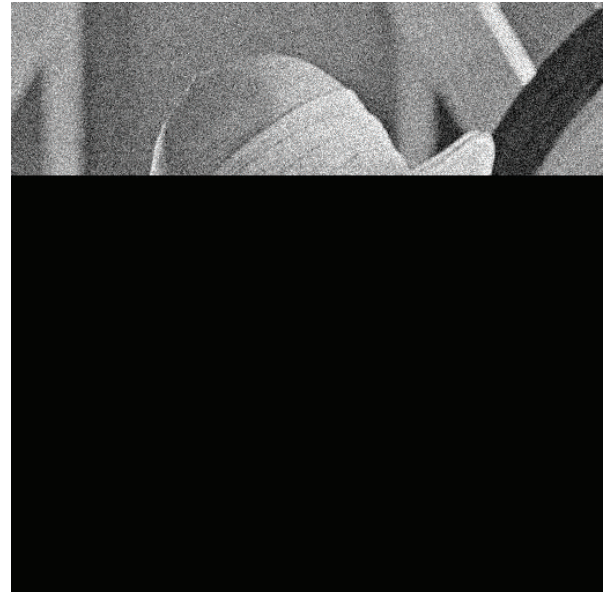

(a)

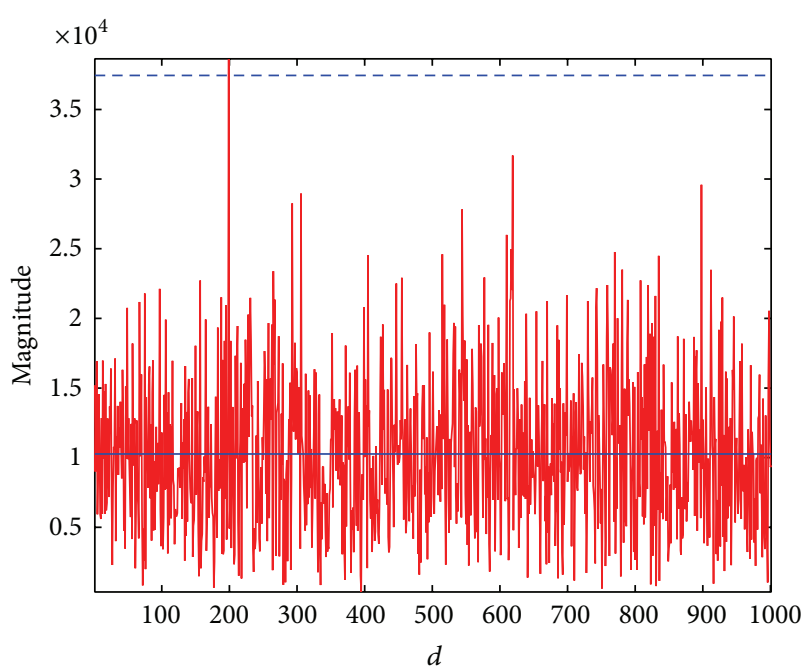

(b)

Figure 8: (a) The upcropped noisy "Lena" of Figure 4(a). (b) The detection of the upcropped noisy "Lena."

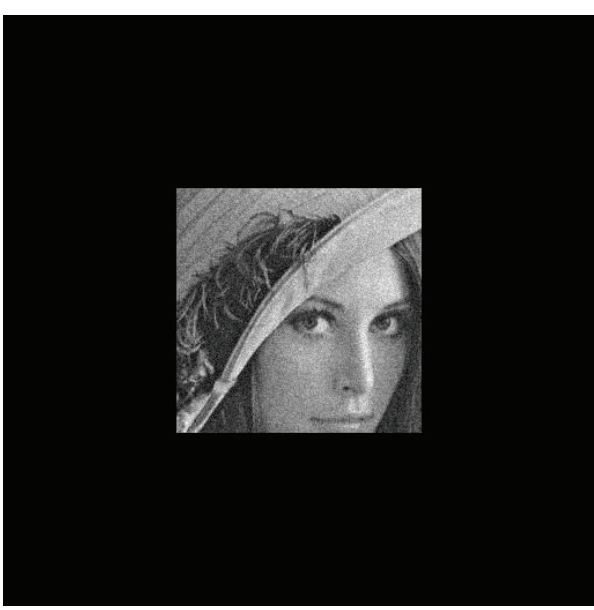

(a)

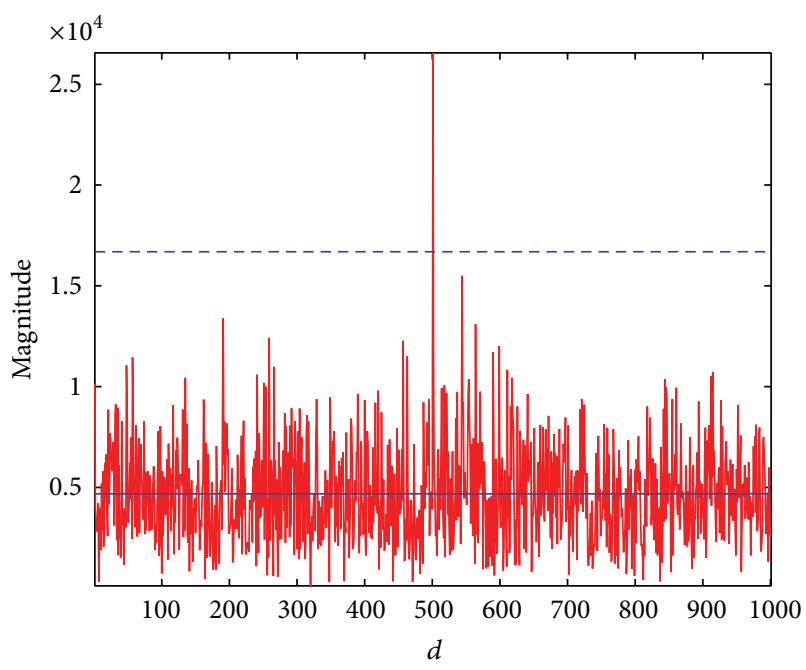

(b)

Figure 9: (a) The central cropped noisy "Lena" of Figure 3(a). (b) The detection of the central cropped noisy "Lena."

the sensitivity of $\gamma_{1}$ is not so gratifying especially when $\gamma_{1}$ is between 0.25 and 0.5 in Figure 11(c).

\section{Conclusion}

A novel watermarking technique based on the discrete LCT is proposed in this paper. In this kind of method, the watermarks are embedded in the middle coefficients in the transform domain, and the detecting threshold is determined adaptively. The simulations for the robustness of the proposed method under the common image processing are performed, and the simulation results fit the theories well. The proposed watermarking is more secure than the watermarking based on FRFT or DCT domain because it has more free parameters. We also discussed the parameter's sensitivity of the proposed method in the paper and showed that this kind of watermarking method is sensitive to the parameters of the LCT.

\section{Conflict of Interests}

The authors declare that there is no conflict of interests regarding the publication of this paper.

\section{Acknowledgments}

This work was supported by the National Natural Science Foundation of China (no. 61072097 and no. 61171195) and 


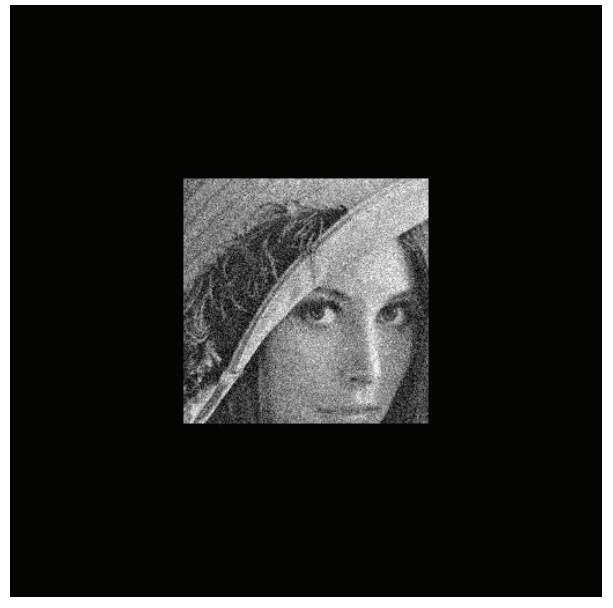

(a)

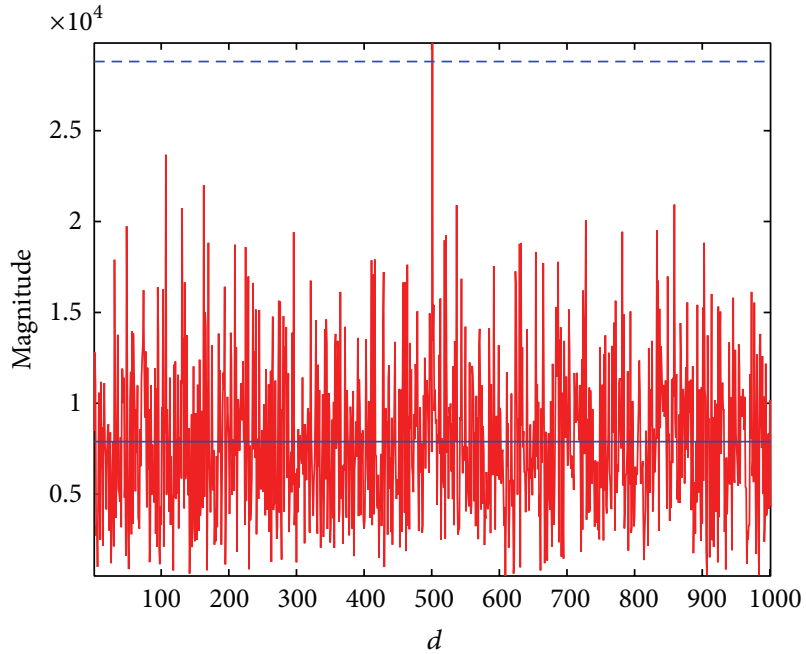

(b)

Figure 10: (a) The central cropped noisy "Lena" of Figure 4(a). (b) The detection of the central cropped noisy "Lena."

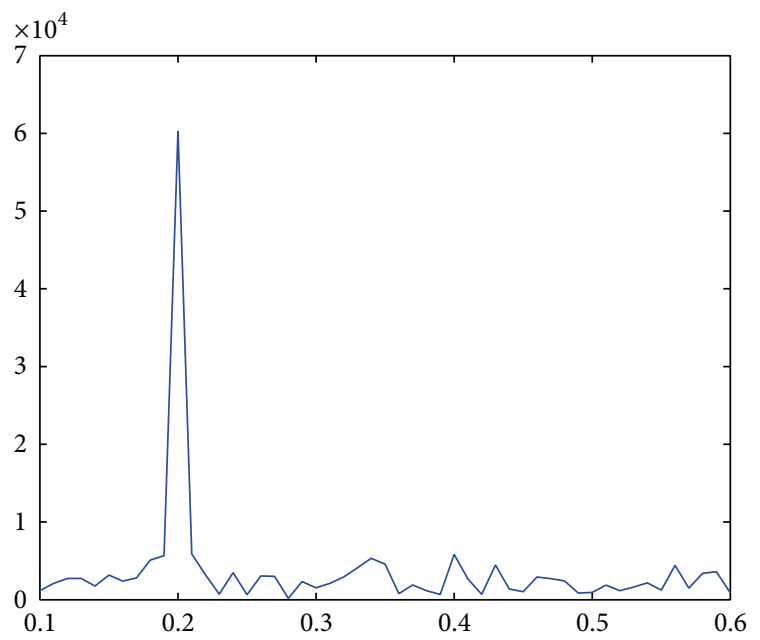

(a)

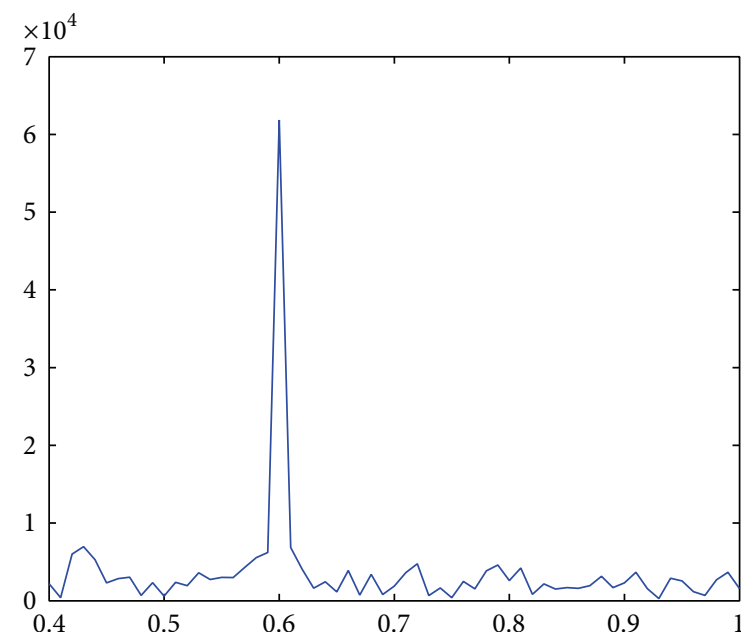

(b)

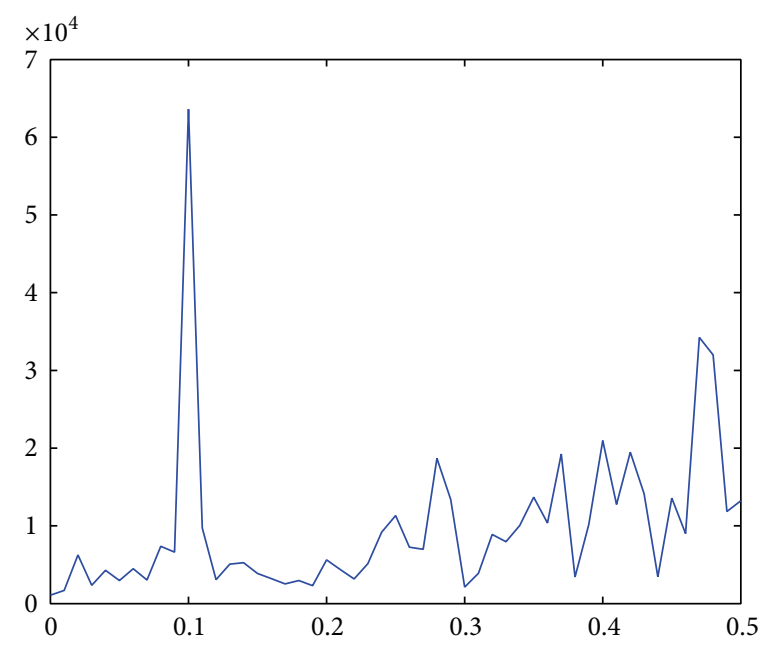

(c)

FIGURE 11: The sensitivity of parameters. (a) The sensitivity of $\alpha_{1}$, (b) the sensitivity of $\beta_{1}$, and (c) the sensitivity of $\gamma_{1}$. 
is also supported by Program for New Century Excellent Talents in University (no. NCET-12-0042).

\section{References}

[1] I. J. Cox, J. Kilian, T. Leighton, and T. Shamoon, "Secure spread spectrum watermarking for images, audio and video," in Proceedings of the IEEE International Conference on Image Processing (ICIP '96), vol. 3, pp. 243-246, Lausanne, Switzerland, September 1996.

[2] K. Eckhard, J. Rindfrey, and J. Zhao, "Copyright protection for multimedia data," in Proceedings of the International Conference on Digital Media and Electronic Publishing, vol. 32, 1994.

[3] I. J. Cox, J. Kilian, F. T. Leighton, and T. Shamoon, "Secure spread spectrum watermarking for multimedia," IEEE Transactions on Image Processing, vol. 6, no. 12, pp. 1673-1687, 1997.

[4] J. J. K. O'Ruanaidh, W. J. Dowling, and F. M. Boland, "Phase watermarking of digital images," in Proceedings of the IEEE International Conference on Image Processing (ICIP '96), vol. 3, pp. 239-242, Lausanne, Switzerland, September 1996.

[5] V. Solachidis and I. Pitas, "Circularly symmetric watermark embedding in 2-D DFT domain," IEEE Transactions on Image Processing, vol. 10, no. 11, pp. 1741-1753, 2001.

[6] S. Liu, B. M. Hennelly, and J. T. Sheridan, "Digital image watermarking spread-space technique based on double random phase encoding," Optical Communications, vol. 300, pp. 162-177, 2013.

[7] M. Barni, F. Bartolini, V. Cappellini, and A. Piva, "A DCTdomain system for robust image watermarking," Signal Processing, vol. 66, no. 3, pp. 357-372, 1998.

[8] A. Piva, M. Barni, F. Bartolini, and V. Cappellini, "DCT-based watermark recovering without resorting to the uncorrupted original image," in Proceedings of the International Conference on Image Processing, vol. 1, pp. 520-523, October 1997.

[9] M. Kutter, "Watermarking world," http://www.watermarkingworld.org/.

[10] C. Candan, M. A. Kutay, and H. M. Ozaktas, "The discrete fractional Fourier transform," IEEE Transactions on Signal Processing, vol. 48, no. 5, pp. 1329-1337, 2000.

[11] H. M. Ozaktas, N. Erkaya, and M. A. Kutay, "Effect of fractional Fourier transformation on time-frequency distributions belonging to the Cohen class," IEEE Signal Processing Letters, vol. 3, no. 2, pp. 40-41, 1996.

[12] H. Ozaktas and D. Mendlovic, "Fractional Fourier transforms and their optical implementation. II," Journal of the Optical Society of America A, vol. 10, no. 12, pp. 2522-2531, 1993.

[13] S.-C. Pei, C.-C. Tseng, M.-H. Yeh, and J.-J. Shyu, "Discrete fractional hartley and fourier transforms," IEEE Transactions on Circuits and Systems II: Analog and Digital Signal Processing, vol. 45, no. 6, pp. 665-675, 1998.

[14] H. M. Ozaktas, M. A. Kutay, and Z. Zalevsky, The Fractional Fourier Transform With Applications in Optics and Signal Processing, Wiley, New York, NY, USA, 2000.

[15] R. Tao, B. Deng, and Y. Wang, Fractional Fourier Transform and Its Applications, University Press, Beijing, China, 2009.

[16] I. Djurovic, S. Stankovic, and I. Pitas, "Digital watermarking in the fractional Fourier transformation domain," Journal of Network and Computer Applications, vol. 24, no. 2, pp. 167-173, 2001.

[17] M. A. Savelonas and S. Chountasis, "Noise-resistant watermarking in the fractional Fourier domain utilizing moment-based image representation," Signal Processing, vol. 90, no. 8, pp. 2521$2528,2010$.

[18] A. Bultheel, "Digital watermarking of images in the fractional Fourier domain," TW Report TW497, 2007.

[19] N. K. Nishchal, "Hierarchical encrypted image watermarking using fractional Fourier domain random phase encoding," Optical Engineering, vol. 50, no. 9, Article ID 097003, 2011.

[20] T.-Z. Xu and B.-Z. Li, Linear Canonical Transform and Its Applications, Science Press, Beijing, China, 2013.

[21] S.-C. Pei and J.-J. Ding, "Closed-form discrete fractional and affine Fourier transforms," IEEE Transactions on Signal Processing, vol. 48, no. 5, pp. 1338-1353, 2000.

[22] A. Koç, H. M. Ozaktas, C. Candan, and M. A. Kutay, "Digital computation of linear canonical transforms," IEEE Transactions on Signal Processing, vol. 56, no. 6, pp. 2383-2394, 2008.

[23] F. S. Oktem and H. M. Ozaktas, "Exact relation between continuous and discrete linear canonical transforms," IEEE Signal Processing Letters, vol. 16, no. 8, pp. 727-730, 2009.

[24] J. J. Healy and J. T. Sheridan, "Sampling and discretization of the linear canonical transform," Signal Processing, vol. 89, no. 4, pp. 641-648, 2009.

[25] B.-Z. Li, R. Tao, and Y. Wang, "New sampling formulae related to linear canonical transform," Signal Processing, vol. 87, no. 5, pp. 983-990, 2007.

[26] A. Stern, "Sampling of linear canonical transformed signals," Signal Processing, vol. 86, no. 7, pp. 1421-1425, 2006.

[27] R. Tao, B.-Z. Li, Y. Wang, and G. K. Aggrey, "On sampling of band-limited signals associated with the linear canonical transform," IEEE Transactions on Signal Processing, vol. 56, no. 11, pp. 5454-5464, 2008.

[28] B.-Z. Li and T.-Z. Xu, "Spectral analysis of sampled signals in the linear canonical transform domain," Mathematical Problems in Engineering, vol. 2012, Article ID 536464, 19 pages, 2012.

[29] B.-Z. Li and T.-Z. Xu, "Sampling in the linear canonical transform domain," Mathematical Problems in Engineering, vol. 2012, Article ID 504580, 13 pages, 2012.

[30] S.-C. Pei and J.-J. Ding, "Eigenfunctions of linear canonical transform," IEEE Transactions on Signal Processing, vol. 50, no. 1, pp. 11-26, 2002.

[31] D. Wei, Q. Ran, Y. Li, J. Ma, and L. Tan, "A convolution and product theorem for the linear canonical transform," IEEE Signal Processing Letters, vol. 16, no. 10, pp. 853-856, 2009.

[32] B. Deng, R. Tao, and Y. Wang, "Convolution theorems for the linear canonical transform and their applications," Science in China. Series F. Information Sciences, vol. 49, no. 5, pp. 592-603, 2006.

[33] J. Zhao, R. Tao, Y.-L. Li, and Y. Wang, "Uncertainty principles for linear canonical transform," IEEE Transactions on Signal Processing, vol. 57, no. 7, pp. 2856-2858, 2009. 


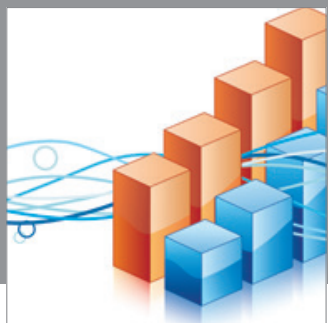

Advances in

Operations Research

mansans

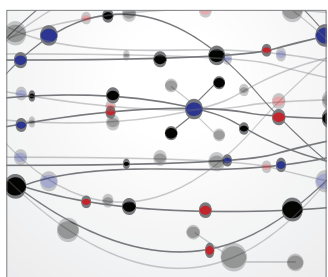

The Scientific World Journal
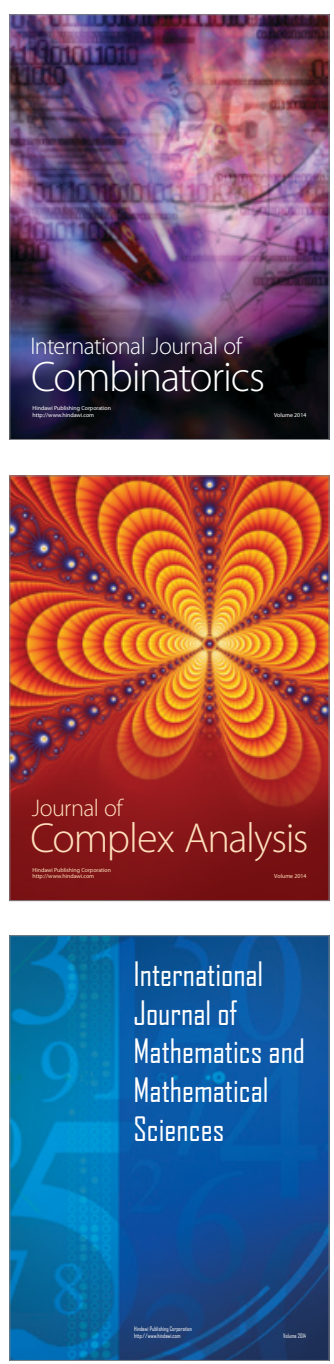
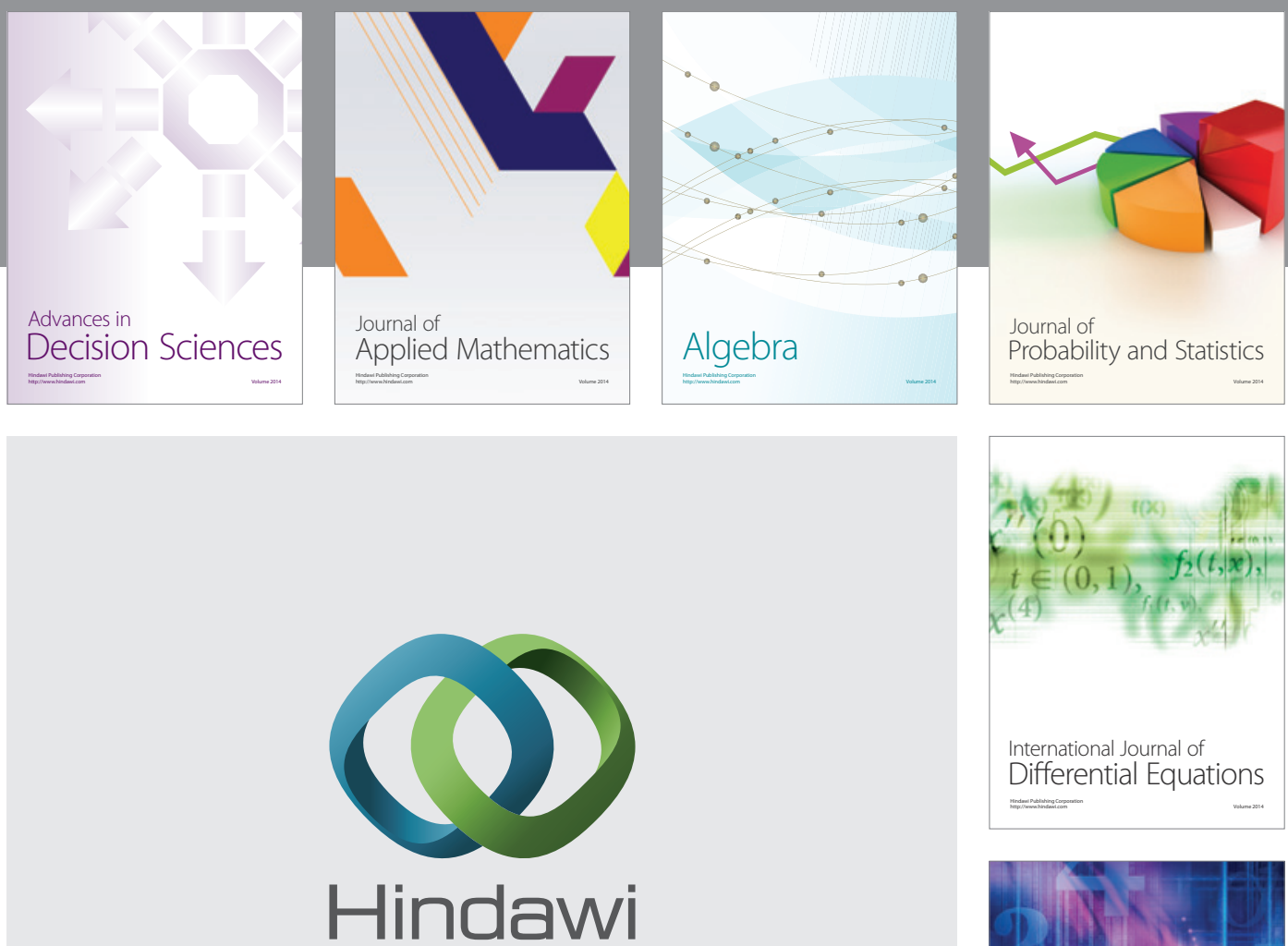

Submit your manuscripts at http://www.hindawi.com
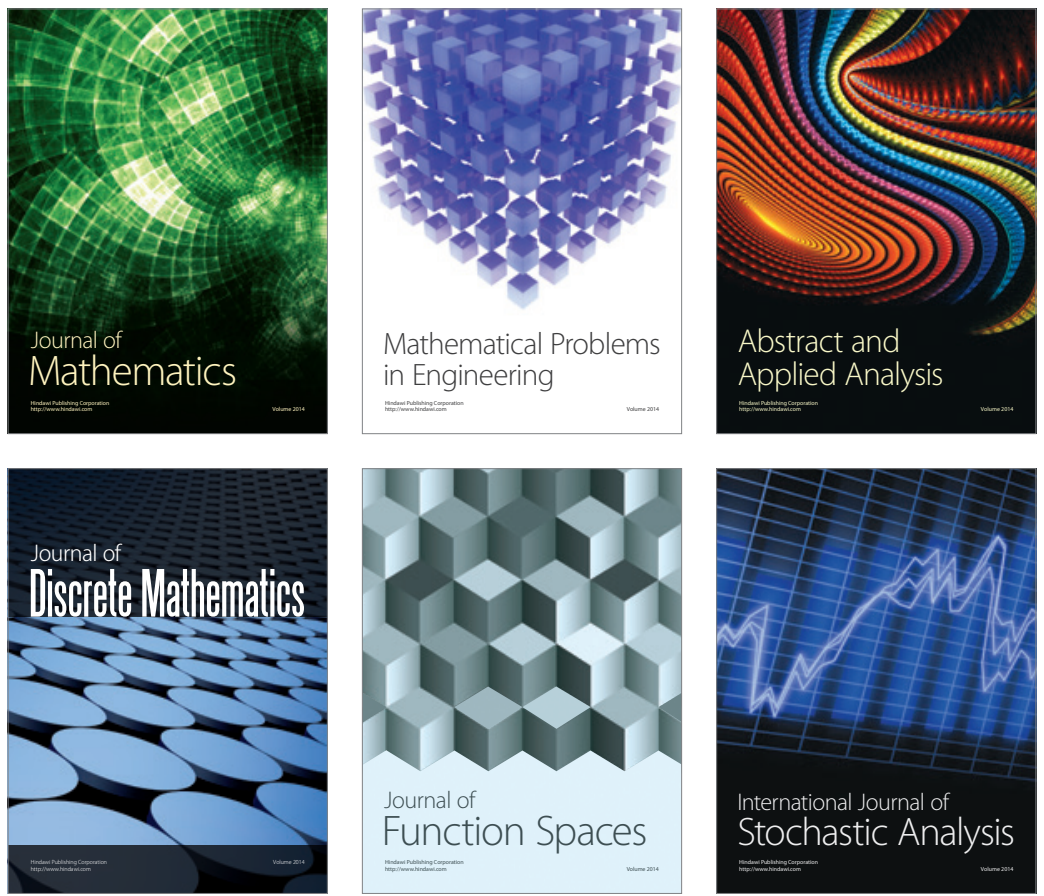

Journal of

Function Spaces

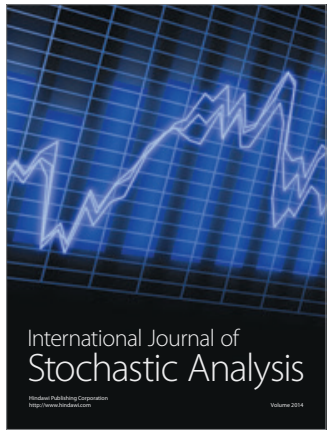

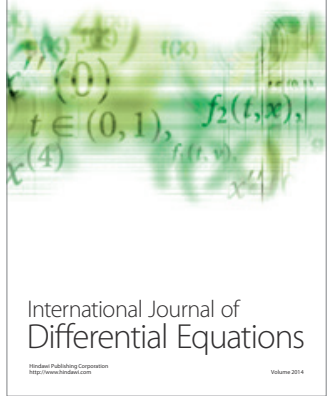
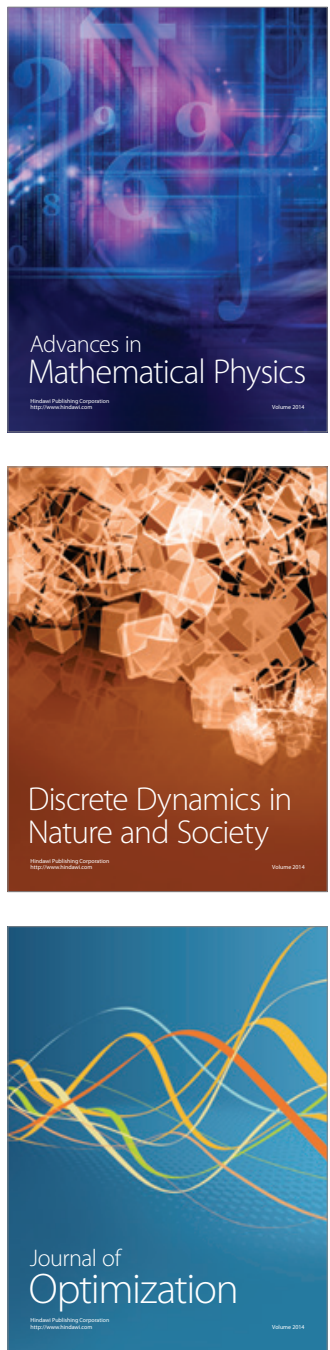